\title{
Olfactory Bulbectomy Impedes Social but Not Photic Reentrainment of Circadian Rhythms in Female Octodon degus
}

\author{
Namni Goel ${ }^{1}$ and Theresa M. Lee \\ Department of Psychology, University of Michigan, Ann Arbor, MI 48109-1109
}

\begin{abstract}
Recent studies demonstrated that nonphotic (social) cues markedly accelerate reentrainment to large phase shifts of the light-dark (LD) cycle in female Octodon degus and that such changes are likely effected by chemosensory stimuli. This experiment investigated the effects of olfactory bulbectomies on (1) socially facilitated reentrainment rates of circadian rhythms following a 6-h phase advance of the LD cycle, (2) photic reentrainment rates of circadian rhythms following a 6-h advance of the LD cycle, (3) photic entrainment, and (4) the circadian period $(\tau)$ of activity rhythms in constant darkness (DD). Olfactory bulbectomies (BX) blocked socially facilitated reentrainment rates but did not alter reentrainment rates of circadian rhythms to photic cues alone. In addition, BX lowered mean daily locomotor activity levels and decreased the amplitude of the activity rhythm in degus housed in entrained (LD 12:12) conditions but did not alter the phase of activity onset or offset, duration $(\alpha)$ of activity, or mean daily core body temperature. Bulbectomies also failed to modify $\tau$ of free-running activity rhythms. This experiment confirms that the olfactory bulbs and chemosensory cues are necessary for socially facilitated reentrainment. In contrast to their effects in nocturnal rodents, BX do not produce significant circadian photic changes in diurnal degus. This is the first experiment to determine that chemosensory stimuli modulate the circadian system in a diurnal rodent.
\end{abstract}

Key words nonphotic, activity, chemosensory, free-running, vomeronasal, diurnal, rodent

\section{INTRODUCTION}

Several recent experiments with Octodon degus, a diurnal South American hystricomorph rodent, demonstrate that social cues affect circadian rhythms in the presence of a light-dark (LD) cycle (Goel and Lee, 1995a, 1995b, 1996). When female "donors" (degus entrained to an LD cycle) are paired continuously with phase-shifted females, their partners reentrain $25 \%$ to $42 \%$ faster than female degus housed alone, following 6-h phase advances (Goel and Lee, 1995a) or delays (Goel and Lee, 1995b). Acceleration is due to social stimuli acting on the circadian system rather than to social masking (Goel and Lee, 1995b); such stimuli are ineffective in phase-shifted males following phase advances or delays (Goel and Lee, 1995a, 1995b).

\footnotetext{
1. To whom all correspondence should be addressed. Present address: New York State Psychiatric Institute/Columbia University, 722 W. 168th Street, Unit 50, New York, NY 10032 and Cornell University Medical Center-New York Hospital, Laboratory of Human Chronobiology, 21 Bloomingdale Rd., White Plains, NY 10605; phone: (212) 543-2469; fax: (212) 543-2467; e-mail: ng129@columbia.edu. Send request for reprints to either address.
}

JOURNAL OF BIOLOGICAL RHYTHMS, Vol. 12 No. 4, August $1997362-370$ (C) 1997 Sage Publications, Inc. 
Cumulative evidence suggests that olfactory information may be the critical social cue necessary for accelerated reentrainment. Not only is accelerated reentrainment of circadian rhythms in the presence of social cues sexually differentiated in degus (Goel and Lee, 1995a, 1995b, 1996), but both the production and attention to olfactory cues (Fischer and Meunier, 1985; Fischer et al., 1986) are also sexually dimorphic, implying that olfaction may be important to phase shifting in this species. Further, Goel and Lee (1996) have shown that auditory and tactile cues do not alter circadian reentrainment; rather, the available data suggest that olfactory cues (in particular, airborne scents) supply the social influence necessary to accelerate reentrainment rates of circadian rhythms in females. Finally, in the absence of light, social cues are sufficient to modulate circadian period $(\tau)$ and phase, resulting in partial entrainment of circadian activity rhythms in female degus housed in constant darkness (DD; Goel and Lee, 1997). Although the critical cue is likely olfactory, we cannot eliminate the role of auditory cues from that study.

Although olfactory stimuli have been associated with nonphotic (social) entrainment in degus, a causal relationship between the importance of chemosensory cues and nonphotic (social) entrainment has not been defined. Thus, we removed the olfactory bulbs to investigate their functional significance on nonphotic (social) reentrainment in degus.

Complete removal of the olfactory bulbs modulates circadian rhythms in nocturnal rodents, albeit the functional importance of these effects remains unknown. Olfactory bulbectomies (BX) lengthen $\tau$ of locomotor activity rhythms in rats (Lumia et al., 1987), hamsters (Pieper and Lobocki, 1991), and mice housed in DD (Possidente et al., 1990, 1996). Bulbectomies delay the phase of activity onset in mice (Possidente et al., 1990, 1996) and hamsters (Bittman et al., 1989; Pieper and Lobocki, 1991) and decrease the nocturnal amplitude of activity rhythms in rats (Lumia et al., 1987). In addition, BX lengthen duration ( $\alpha$ ) of activity in hamsters (Pieper and Lobocki, 1991), increase daily mean activity levels in mice housed in LD or DD (Possidente et al., 1996), and alter LD activity ratios in rats (Lumia et al., 1992). Thus far, bulbectomy studies have not been performed in a diurnal rodent to assess circadian changes. Therefore, the second purpose of this experiment was to determine the effect of olfactory bulbectomy removal on photic entrainment and on free-running circadian rhythms in a diurnal species.

\section{MATERIALS AND METHODS}

\section{Subjects}

Subjects were 13 mature female $O$. degus ( 1 to 2 years of age, with an average life span of 5-7 years) born in a laboratory colony at the University of Michigan. Prior to this experiment, animals were housed in LD 12:12, lights on at $0600 \mathrm{~h}$ (average light intensity was 250 lux), with room temperature at $21^{\circ} \mathrm{C} \pm 2^{\circ} \mathrm{C}$ and humidity held at $50 \%$ to $60 \%$. Degus were maintained on a diet of Purina Rodent Chow and Guinea Pig Chow supplemented weekly with apples and peanuts. Both food and water were available ad libitum.

\section{Housing}

During the entrained and photic reentrainment phases of the experiment, females were housed individually in $42.5 \times 22 \times 19 \mathrm{~cm}$ cages fitted with Nalgene running wheels ( $9 \mathrm{~cm}$ wide $\times 34.5 \mathrm{~cm}$ in diameter) in LD 12:12 in the room conditions noted above. During the socially facilitated reentrainment phase, both individuals in each social pair were housed with running wheels in plastic cages $(42.5 \times 46 \times 19.5 \mathrm{~cm})$ with a wire mesh divider placed in the middle. The divider prevented physical contact between animals on opposite sides of the mesh (e.g., aggression or touching) but allowed visual, olfactory, and auditory exchange (Goel and Lee, 1995a, 1995b, 1996). All pairs were composed of unrelated, unfamiliar female conspecifics. Finally, in DD (0 lux), females were again housed individually with running wheels in conditions as noted above.

\section{Data Collection}

Running wheel activity and core body temperature data were collected, recorded, and stored in 10-min bins by Dataquest III (Mini-mitter Co., Sunriver, OR). Mini-mitter transmitters (Model VM-FH Disc; Mini-mitter Co., Sunriver, OR) were used to monitor core body temperature.

\section{Procedure}

Transmitters were surgically implanted in the peritoneal cavity of each animal. Degus were anesthetized with Xylazine (i.p., $4 \mathrm{mg} / \mathrm{kg}$ body weight) and Ketamine HCL (i.p., $120 \mathrm{mg} / \mathrm{kg}$ body weight). Yohimbine (i.p., 4 
Pre-SHAM/BX entrained phase ( 3 weeks)

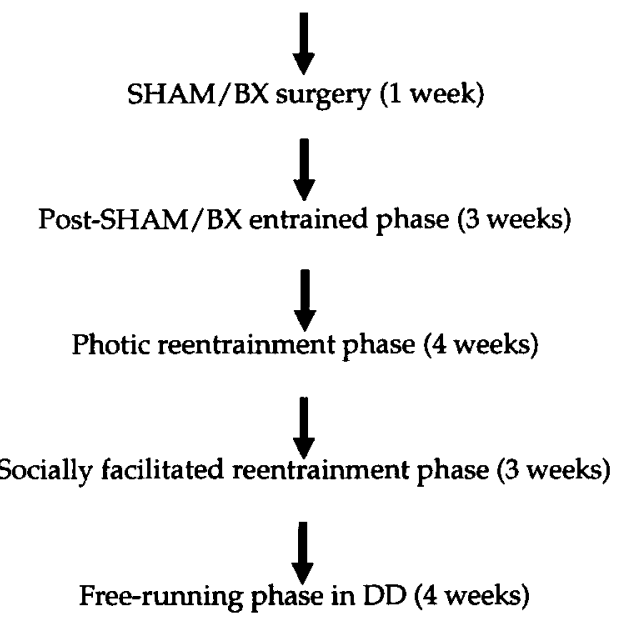

Figure 1. A diagrammatic representation of the different experimental phases. The number of weeks degus spent in each condition is indicated in parentheses. See text for details.

$\mathrm{mg} / \mathrm{kg}$ body weight) and $5 \mathrm{cc}$ lactate ringers (s.c.) were administered immediately after surgery to facilitate recovery.

Degus were housed in LD 12:12, lights on at $1200 \mathrm{~h}$, for 3 weeks following postoperative recovery from transmitter surgery (pre-SHAM/BX entrained phase; Fig. 1). Next, animals underwent either sham surgery or olfactory bulbectomies (described below) and remained in LD 12:12, lights on at $1200 \mathrm{~h}$, for 3 additional weeks following surgical procedures (postSHAM/BX entrained phase; Fig. 1). The LD cycle was then advanced $6 \mathrm{~h}$ (a single shortening of the light phase; darkness began at $1800 \mathrm{~h}$ on the day of the advance), and experimental animals remained in the new LD cycle (LD 12:12, lights on at $0600 \mathrm{~h}$ ) for 4 weeks to ensure complete reentrainment (photic reentrainment phase; Fig. 1). All degus were then transferred from their individual cages to larger cages that were divided by mesh barriers and equipped with Nalgene running wheels on either side (socially facilitated reentrainment phase; Fig. 1). The experimental animals were again phase advanced $6 \mathrm{~h}$ to LD 12:12, lights on at $2400 \mathrm{~h}$ (a single shortening of the light phase; darkness began at $1200 \mathrm{~h}$ on the day of the advance); each experimental animal was placed on one side of a divided cage with an unfamiliar female degu on the other side that had been entrained to this LD cycle for 3 weeks prior to pairing (social "donor"; Goel and Lee, 1995a, 1995b, 1996). The degus remained paired together for 3 weeks to ensure complete reentrainment of the phase-shifting animals' circadian rhythms. Finally, experimental degus were released into DD, where they remained for 4 weeks (free-running phase; Fig. 1).

\section{Surgery}

Degus were randomly assigned to either the SHAM $(n=5)$ or BX $(n=8)$ group and were anesthetized with $120 \mathrm{mg} / \mathrm{kg}$ Ketamine HCL (i.p.), $4 \mathrm{mg} / \mathrm{kg}$ Xylazine (i.p.), and $.1 \mathrm{mg} / \mathrm{kg}$ Butorphanol (i.p.). Sham animals were positioned in a Kopf stereotaxic, and a 2-cm hole was drilled through the skull bilaterally, lateral to the midline, and just dorsal to the anterior tip of the eye. An identical procedure was used for bulbectomies, except the olfactory bulbs were ablated via an aspiration Suction Vac (Schuco, Inc., Toledo, OH). Tissue dorsal to the cribiform plate and rostral to the frontal poles was removed, but the superior sagittal sinus was left intact. Bilateral bulbectomies were accomplished by performing unilateral aspirations separated by a 3to 5-day recovery period. A two-step surgery procedure was implemented because attempts at one-stage bilateral aspirations invariably resulted in death immediately after surgery. Following surgery, degus received $4 \mathrm{mg} / \mathrm{kg}$ Yohimbine (i.p.), $2 \mathrm{mg} / \mathrm{kg}$ Dexamethasone (s.c.), .2 mg/kg Butorphanol (s.c.), and $10 \mathrm{cc}$ lactated ringers (s.c.) to facilitate recovery.

\section{Data Analysis}

Six measures were analyzed using daily histograms for data collected in entrained conditions (pre- and post-SHAM/BX entrained phases): activity onset, activity offset, $\alpha$ of activity, amplitude of activity, mean daily activity level, and mean daily core body temperature. For each measure, 10 to 12 days of entrained data were averaged to determine a mean value per animal. The measures were defined as follows: activity onset was the point when 40 counts of activity per 10 min for at least a 20-min period occurred following a minimum 4-h hiatus of activity (Goel and Lee, 1995a, 1995b); activity offset was the point when locomotor activity dropped below the daily mean for at least 4 hours (Labyak et al., 1997; Lee and Labyak, 1997); $\alpha$ of activity was the difference between activity onset and 


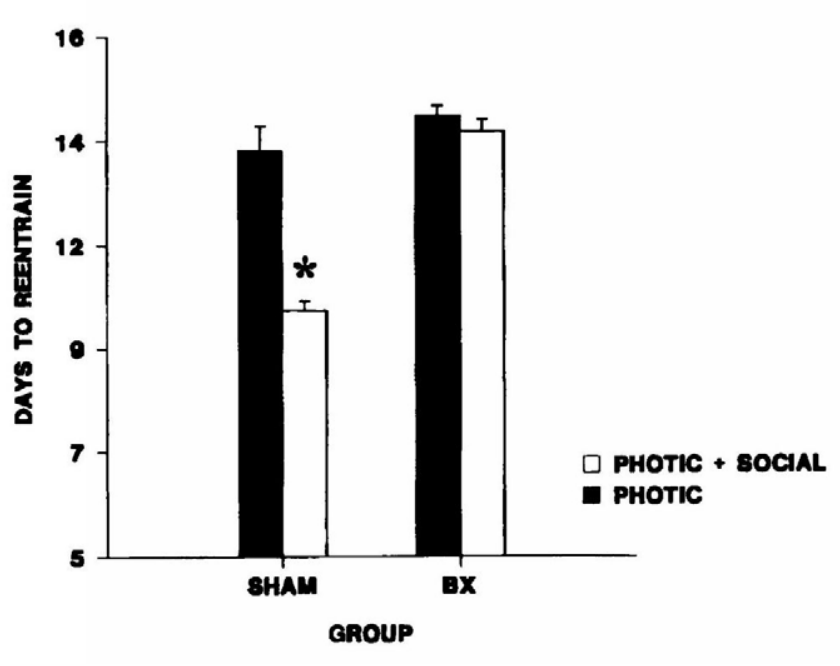

Figure 2. The mean ( $\pm S E M)$ number of days required for reentrainment of the locomotor activity rhythm to a 6-h advance of the LD cycle with and without nonphotic (social) cues. Symbols: SHAM $=$ sham surgery animals $(n=5) ; B X=$ bulbectomized animals $(n=6) .{ }^{*}=$ significantly less than the BX group, $p<.001$.

offset; and amplitude of the activity rhythm was the difference between the daily rhythm peak (maximum value in a 10-min data bin) and the daily mean (Goel and Lee, 1995a; Labyak and Lee, 1995). Mean daily activity and mean daily temperature were calculated from midnight each day for the entire 24-h interval; means for both measures were calculated from data collected at identical 10-min intervals.

Following the photic and socially facilitated phase advances, daily histograms and actograms of temperature and activity data were used to determine the reentrainment rate (the number of days) it took for each animal's activity and temperature rhythms to reestablish their former phase relationships to the new lighting schedule (Goel and Lee, 1995a, 1995b, 1996). Reentrainment was assessed for the temperature rhythm by monitoring the daily temperature rise following the daily temperature minimum. Temperature minimum is the time of the lowest temperature of the day and occurs during the temperature trough approximately $3 \mathrm{~h}$ prior to lights on in entrained animals (Goel and Lee, 1995a, 1995b, 1996). Reentrainment of the activity rhythm was evaluated by monitoring activity onset (defined above). In previous work, we have demonstrated that the above method is a reliable measure of socially facilitated reentrainment, as opposed to masking, of the circadian system; the activity rhythms of degus placed into DD the day immediately following completion of socially facilitated reentrainment to a 6-h phase advance began free-running from the determined phase of reentrainment (Goel and Lee, 1995b).

The free-running $\tau$ of locomotor activity rhythms for the last 2 weeks of data collected in DD was determined by Cosine analysis and corroborated by Fourier analysis (Dataquest). Multivariate repeated measures analysis of variance and post hoc comparisons using Bonferroni-adjusted probabilities were used to compare reentrainment rates and locomotor activity levels across reentrainment conditions and between groups and to compare prebulbectomy- and postbulbectomyentrained circadian measures. One-way analysis of variance compared differences between groups in $\tau$ and locomotor activity levels in DD. Data are presented as mean \pm SEM where applicable, and $p<.05$ was considered significant.

\section{RESULTS}

\section{Bulbectomy Verification}

The extent of neural damage was assessed via gross examination of the brains under a dissecting microscope. In 6 of the $8 \mathrm{BX}$ degus, the olfactory bulbs were completely destroyed; 2 of these animals also had slight bilateral frontal lobe damage. The remaining 2 animals sustained partial bilateral bulbectomies. Using microscopic examination, cresyl violet-stained coronal and sagittal sections from the partial animals were compared with similar sections from two SHAM animals. In both of the partially damaged brains, normal appearing portions of the main olfactory bulbs (MOB) were evident, but the accessory olfactory bulbs (AOB) and the vomeronasal organ (VNO) were absent. Data from the partial BX group were not included in the statistical analyses.

\section{Socially Facilitated Reentrainment}

The locomotor activity rhythms of the SHAM group reentrained faster after a 6 -h phase advance of the LD cycle in the presence of social and photic cues than those of the BX group (Figs. 2 and 3; $p<.001$ ). In addition, the SHAM group's rhythms reentrained faster in the presence of social cues and photic cues 
A

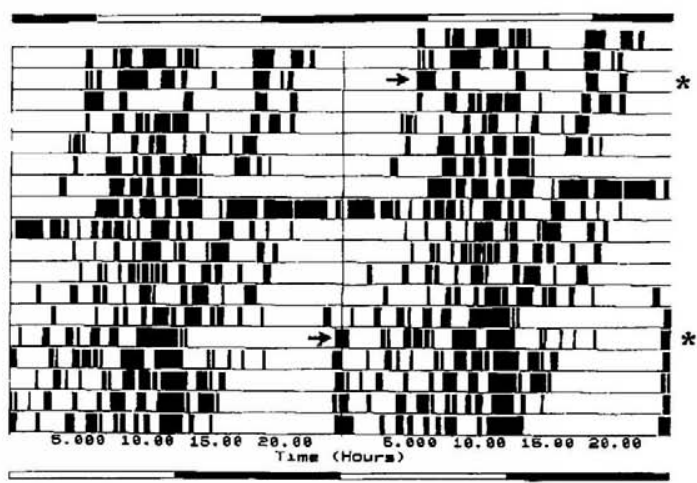

B

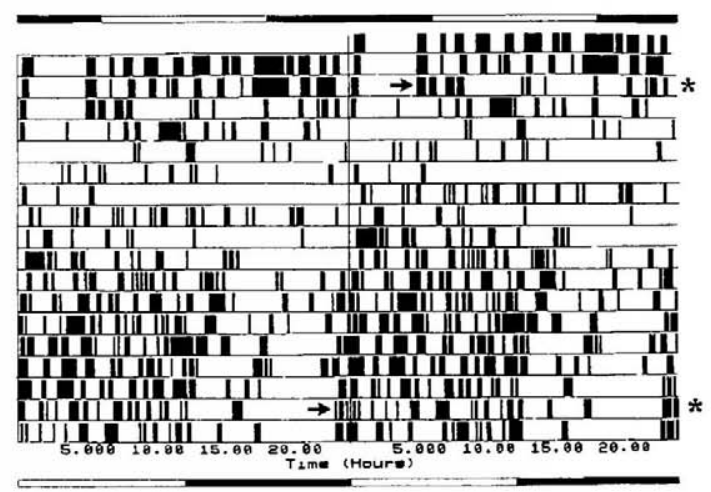

Figure 3. Representative double-plotted actograms depicting reentrainment rates of the circadian locomotor rhythm to both social and photic cues for a SHAM female (A) and a BX female (B). Each line represents one day of data, with the lowest $10 \%$ of activity values cut from the figure for ease in viewing activity onset. The top LD bar indicates the initial lighting schedule (LD 12:12, lights on at $0600 \mathrm{~h}$ ). The first asterisk in the right margin marks the day of the phase shift (6-h advance), and the first arrow denotes the phase angle prior to the shift. The bottom LD bar indicates the new lighting schedule (LD 12:12, lights on at $2400 \mathrm{~h}$ ). The second asterisk in the right margin denotes the day of reentrainment, as determined by the reestablished phase angle (second arrow). In (A), the sustained increase in locomotor activity during Days 5 through 6 (following the phase shift) typifies behavioral estrus in adult females (Labyak and Lee, 1995).

compared with photic cues alone (Figs. 2,3, and 4; $p<$ $.003)$, while no significant reentrainment rate differences were found between entrainment conditions for the BX group (Figs. 2, 3, and 4). Socially facilitated reentrainment rates of the core body temperature rhythm were virtually identical to those for the locomotor activity rhythm, as reported previously (Goel and Lee, 1995a, 1995b, 1996), and are therefore not
A

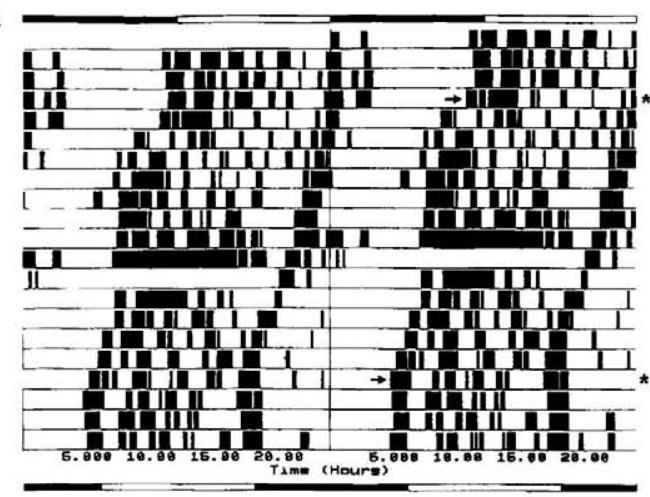

B

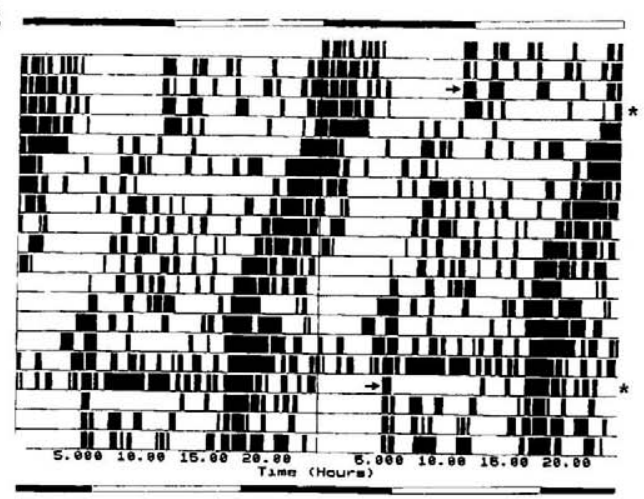

Figure 4. Representative double-plotted actograms depicting reentrainment rates of the circadian locomotor rhythm to photic cues alone for a SHAM female (A) and a BX female (B). Each line represents one day of data, with the lowest $10 \%$ of activity values cut from the figure for ease in viewing activity onset. The top LD bar indicates the initial lighting schedule (LD 12:12, lights on at $1200 \mathrm{~h}$ ). The first asterisk in the right margin marks the day of the phase shift (6-h advance), and the first arrow denotes the phase angle prior to the shift. The bottom LD bar indicates the new lighting schedule (LD 12:12, lights on at $0600 \mathrm{~h}$ ). The second asterisk in the right margin denotes the day of reentrainment, as determined by the reestablished phase angle (second arrow).

described in detail. The groups did not significantly differ in mean daily activity levels (number of wheel revolutions $/ 10 \mathrm{~min}$ ) for the first 3 days of the phase shift, nor did their activity levels change significantly across these days.

\section{Photic Reentrainment}

There were no significant differences between the SHAM and BX groups in the reentrainment rates of circadian rhythms to photic cues alone (Figs. 2 and 4). As was true for socially facilitated reentrainment, 

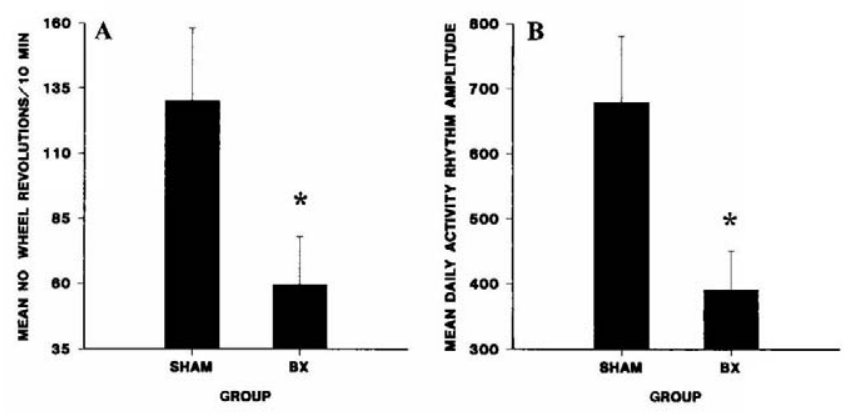

Figure 5. The mean ( \pm SEM) daily activity levels (A) and daily amplitude (B) of locomotor circadian activity rhythms for SHAM $(n=5)$ and bulbectomized $(B X ; n=6)$ animals housed in LD 12:12, lights on at $1200 \mathrm{~h}$ for 3 weeks. Mean values were derived from data collected during the last 10 to 12 days in entrained conditions. Symbols as in Figure 2. * = significantly less than the SHAM group, $p<0.05$.

photic reentrainment rates of the core body temperature rhythm were virtually identical to those for the locomotor activity rhythm, again confirming previous reports (Goel and Lee, 1995a, 1995b, 1996). In addition, there were no significant differences between the SHAM and BX groups in mean daily locomotor activity levels (number of wheel revolutions $/ 10 \mathrm{~min}$ ) for the first 3 days of the phase shift; however, both groups had decreased locomotor activity levels on the first day after the shift compared with the day of the shift and the second day after the shift $(p<.02)$.

\section{Entrained Conditions}

The BX and SHAM groups did not differ significantly in the phase of activity onset or offset, $\alpha$ or amplitude of the activity rhythm, or daily mean locomotor activity or core body temperature for the last 10 to 12 days in entrained conditions prior to SHAM/BX surgery. However, during the last 10 to 12 days (of a 3-week period) spent in entrained conditions following SHAM/BX surgery, BX animals had lower mean daily locomotor activity levels than SHAM animals (Fig. 5A; $p<.04$ ) and had a decreased amplitude of the locomotor activity rhythm (Fig. $5 \mathrm{~B} ; p<.02$ ). The two groups did not differ significantly in the phase of activity onset (SHAM: $-1.13 \pm 2.19 \mathrm{~h}$; BX: $+0.44 \pm 0.43$ $h ; p=.44$ ), phase of activity offset (SHAM: $-16.45 \pm 1.29$ $h$; BX: $-14.54 \pm 0.64 h ; p=.18$ ); $\alpha$ of activity (SHAM: $15.29 \pm 1.10 \mathrm{~h} ; \mathrm{BX}: 15.32 \pm 0.65 \mathrm{~h} ; p=.98$ ), or daily mean core body temperature (SHAM: $37.75 \pm 0.27^{\circ} \mathrm{C}$; $\mathrm{BX}$ : $37.55 \pm 0.22^{\circ} \mathrm{C} ; p=.58$ ).

\section{Free-Running Conditions}

The groups did not differ significantly in $\tau$ of freerunning activity rhythms in DD (SHAM: $23.18 \pm 0.13 \mathrm{~h}$; BX: $23.26 \pm 0.10 h ; p=.67$ ) or in mean daily locomotor activity levels (number of wheel revolutions $/ 10 \mathrm{~min}$ ) while in DD (SHAM: 137.62 \pm 24.87 ; BX: $120.92 \pm 26.38$; $p=.65)$.

\section{DISCUSSION}

Removal of the olfactory bulbs prevented socially facilitated reentrainment of circadian rhythms but did not alter reentrainment rates to photic cues alone or modify $\tau$ of free-running activity rhythms in DD. Olfactory bulbectomies reduced mean daily locomotor activity levels and decreased the amplitude of the locomotor activity rhythm in entrained conditions but did not modify the phase of activity onset or offset, $\alpha$ of the activity rhythm, or mean core body temperature. Thus, the olfactory bulbs and chemosensory cues are necessary for the induction of socially facilitated reentrainment of circadian rhythms. In contrast, olfactory bulbectomies were without effect on photic circadian measures in diurnal degus, which differs from previous reports in other rodent species.

Reentrainment of circadian rhythms in the presence of combined social and photic cues was blocked by olfactory bulbectomies, providing strong evidence that chemosensory cues mediated through these structures are critical for socially facilitated reentrainment in female degus. Furthermore, the data from two animals with incomplete bulbectomies indicate that even partial damage to the olfactory bulbs was sufficient to affect socially facilitated entrainment, as the reentrainment rates of these animals' rhythms were intermediate to those of the complete BX and SHAM animals.

In addition to regulating circadian rhythms, chemosensory cues are essential for other behaviors in degus. For example, scent marking is an integral part of degu burrow marking (Fulk, 1976) and agonistic interactions (Davis, 1975). Like degus, golden hamsters can use olfactory cues to modulate circadian rhythms: olfactory cues emitted by females in estrus increase reentrainment rates of rhythms following 
phase shifts (Honrado and Mrosovsky, 1989) and induce phase shifts of the activity rhythm in the presence of an LD cycle in males (Honrado and Mrosovsky, 1991).

Chemosensory stimuli must influence reentrainment through the vomeronasal organ and/or main olfactory bulbs. At present, the experimental procedures used in this and previous studies (Goel and Lee, 1995a, 1995b, 1996) do not allow us to distinguish between the functional roles of these two structures or the likelihood that the critical cues are volatile or nonvolatile. However, analysis of the tissue remnants from the two partially bulbectomized animals suggests several possibilities. First, the finding that the accessory olfactory bulbs, and therefore the VNO system, were not intact in the partial BX animals suggests that the VNO system may be important for mediating chemosensory information to the "clock." Second, because the main olfactory system appeared to be only partially functional in these animals, some deficits in reentrainment may be due to a loss of input from this system. Finally, the two systems may collectively mediate chemosensory information and contribute to the slower reentrainment observed in the BX group compared with the SHAM group. Data from more than two animals are needed to distinguish among such possibilities.

Differences in socially facilitated reentrainment rates cannot be attributed to differences in activity levels during the phase-shifting period because the groups did not vary in mean locomotor activity levels for the first 3 days of the shift. This result was unexpected, as our previous research predicted that the SHAM group would have higher activity levels than the BX group due to increases in investigatory-related behaviors (Goel and Lee, 1996). These findings may be explained by considering that both groups had lower mean locomotor activity levels in the socially facilitated condition compared with the photic condition, perhaps as a result of engaging in (or attempting to engage in) olfactory investigation at the barrier (Goel and Lee, 1996) instead of running vigorously in wheels. Thus, the present results are consistent with other data from degus in which the phase shifting of body temperature or activity rhythms was not associated with increases in activity (Goel and Lee, 1995a, 1995b, 1996).

Although activity levels during reentrainment were not altered in BX animals, neural insult resulting from bulbectomies may have affected other behavioral variables, such as fear or avoidance of conspecifics, which in turn could account for the lack of socially facilitated reentrainment effects. To rule out other possibilities, additional experiments involving more selective olfactory damage should be performed. One such example would be to measure socially facilitated reentrainment rates in female degus following olfactory deafferentation with zinc sulfate.

Our data indicate that the olfactory bulbs do not affect photic entrainment per se in degus. Bulbectomies did not alter $\alpha$ or activity onset, in contrast to reports in hamsters and mice (Bittman et al., 1989; Pieper and Lobocki, 1991; Possidente et al., 1990, 1996), nor did they change the distribution of activity in degus, a finding that differs from data from rats (Lumia et al., 1992). Bulbectomies decreased activity levels, an opposite effect from that reported in mice (Possidente et al., 1996); however, BX decreased the amplitude of the activity rhythm, consistent with reports in rats (Lumia et al., 1987). Finally, bulbectomies were without effect on the phase of activity offset, which appears to be delayed in mice (B. Possidente, personal communication), and on reentrainment rates of circadian rhythms to photic cues alone. Notably, all other bulbectomy circadian studies have been performed using male nocturnal rodents, and thus the species differences reported here could be due to a sex difference or a difference between nocturnal and diurnal rodents. Other factors-including differences in experimental procedures, use of small sample sizes, and variations in definitions of circadian variables among studiesmay also underlie the species difference reported here.

Olfactory bulbectomies did not alter $\tau$ of activity rhythms in degus. This differs from rats, hamsters, and mice, in which $\tau$ was lengthened following bulbectomy (Lumia et al., 1987; Pieper and Lobocki, 1991; Possidente et al., 1990, 1996). Once again, such interspecies variation could be due to sex, nocturnal/diurnal, or procedural differences.

Our data imply a dissociation between photic and nonphotic (social) afferent routes to the suprachiasmatic nuclei (SCN) in degus. Because photic entrainment and reentrainment of circadian rhythms remained normal following bulbectomies, the photic entrainment pathway likely is not disrupted following this type of neural damage. In degus, photic information is presumed to be transduced to the $\mathrm{SCN}$ via the retinohypothalamic tract (RHT) and secondarily by the geniculohypothalamic tract (GHT), and both pro- 
ject bilaterally to the SCN (Goel, 1996). In contrast, socially facilitated reentrainment is hindered by $\mathrm{BX}$, suggesting that a distinct pathway transmits social (i.e., chemosensory) information to the SCN.

Two possible neural pathways by which olfactory information could reach the SCN are via (1) the stria terminalis or (2) the retinotelencephalic tract. Lesions of the stria terminalis deplete neuropeptide Y (NPY), metenkephalin (MENK), vasoactive intestinal polypeptide (VIP), and somatostatin (SS) immunoreactive fibers in the SCN in rats (Allen et al., 1984; Palkovits et al., 1981a, 1981b), indicating that one or more of the stria terminalis fiber targets contains cells immunoreactive for these neurotransmitters that project to the SCN. One such target may be the bed nucleus of the stria terminalis (BNST), which projects directly to the SCN (hamsters; Pickard, 1982) and receives projections from the AOB (reviewed in Halpern, 1987).

Alternately, the olfactory tubercle may serve as a point of sensory convergence of visual and chemosensory information and may be involved in the circadian control of behavior in mammals. The retinotelencephalic tract projects from the retina to the olfactory tubercle and appears to be a consistent feature in all mammalian orders (Cooper et al., 1989, 1994; Mick et al., 1993; Tessonneaud et al., 1994). In addition, the olfactory tubercle receives projections from the olfactory bulbs (Cooper et al., 1994; De Olmos et al., 1978; Scalia and Winans, 1975; Scott et al., 1980) and is part of the neural pathway that mediates the increase in gonadotropin secretion found in male BX hamsters (Gomez et al., 1996). To date, however, work on the efferents of the olfactory tubercle does not provide an obvious explanation for the possible sources of control of the SCN.

This experiment demonstrates that chemosensory stimuli can markedly modify the circadian system and represents a novel example of the elimination of a specific sensory nonphotic zeitgeber by neural damage in a diurnal rodent. It should now be possible to determine how and where this sensory information influences "clock" function in degus-whether it be at the SCN or via an interactive site that synapses on the $\mathrm{SCN}$. This study also provides provocative evidence that rodent species may differ in the neural structures, pathways, and specific cues used to transduce nonphotic information to the SCN and thereby regulate circadian rhythms; such knowledge may ultimately lead to an understanding of the adaptive significance of nonphotic zeitgebers.

\section{ACKNOWLEDGMENTS}

We thank Sarah Winans Newman for her assistance in assessing tissue damage in the BX degus and for her comments on a previous version of this manuscript. We also thank Kaye Brabec (Morphology Core in the P30 Center for the Study of Reproduction, University of Michigan) for preparing the tissue from the partial BX animals. This research was funded by NIMHGrant MH49089 to Theresa M. Lee.

\section{REFERENCES}

Allen YS, Roberts GW, Bloom SR, Crow TJ, and Polak JM (1984) Neuropeptide $Y$ in the stria terminalis: Evidence for an amygdalofugal projection. Brain Res 321:357-362.

Bittman EL, Crandell RG, and Lehman MH (1989) Influence of the paraventricular and suprachiasmatic nuclei and olfactory bulbs on melatonin responses in the golden hamster. Biol Reprod 40:118-126.

Cooper HM, Mick G, and Magnin M (1989) Retinal projection to mammalian telencephalon. Brain Res 477:350-357.

Cooper HM, Parvopassu F, Herbin M, and Magnin M (1994) Neuroanatomical pathways linking vision and olfaction in mammals. Psychoneuroendocrinology 19:623-639.

Davis TM (1975) Effects of familiarity on agonistic encounter behavior in male degus (Octodon degus). Behav Biol 14:511-517.

De Olmos J, Hardy H, and Heimer L (1978) The afferent connections of the main and the accessory olfactory bulb formations in the rat: An experimental HRP-study. J Comp Neurol 181:213-244.

Fischer RB and Meunier GF (1985) Responses to conspecifics' urine by the degu (Octodon degus). Physiol Behav 34:99101.

Fischer RB, Smith SL, White PJ, and Meunier GF (1986) Sex differences during initial social contact in the degu (Octodon degus). Behav Process 12:67-76.

Fulk GW (1976) Notes on the activity, reproduction, and social behavior of Octodon degus. J Mammal 57:495-505.

Goel N (1996) Nonphotic entrainment of circadian rhythms in the diurnal rodent, Octodon degus: Behavioral and neuroanatomical integration. Ph.D. dissertation, Department of Psychology, University of Michigan.

Goel N and Lee TM (1995a) Sex differences and effects of social cues on daily rhythms following phase advances in Octodon degus. Physiol Behav 58:205-213.

Goel N and Lee TM (1995b) Social cues accelerate reentrainment of circadian rhythms in diurnal female Octodon 
degus (Rodentia-Octodontidae). Chronobiol Int 12:311323.

Goel N and Lee TM (1996) Relationship of circadian activity and social behaviors to reentrainment rates in diurnal Octodon degus (Rodentia). Physiol, Behav 59:817-826.

Goel N and Lee TM (1997) Social cues modulate free-running circadian activity rhythms in the diurnal rodent, Octodon degus. Am J Physiol, forthcoming.

Gomez DM, Newman SW, and Pieper DR (1996) Lesions of the ventral striatum mimic the effect of olfactory bulbectomy to prevent short photoperiod-induced testicular regression in golden hamsters. Brain Res 723:148-153.

Halpern M (1987) The organization and function of the vomeronasal system. Ann Rev Neurosci 10:325-362.

Honrado GI and Mrosovsky N (1989) Arousal by sexual stimuli accelerates the re-entrainment of hamsters to phase advanced light-dark cycles. Behav Ecol Sociobiol 25:57-63.

Honrado GI and Mrosovsky N (1991) Interaction between periodic socio-sexual cues and light-dark cycles in controlling the phasing of activity rhythms in golden hamsters. Ethol Ecol Evol 3:221-231.

Labyak SE and Lee TM (1995) Estrus- and steroid-induced changes in circadian rhythms in a diurnal rodent, Octodon degus. Physiol Behav 58:573-585.

Labyak SE, Lee TM, and Goel N (1997) Rhythm chronotypes in a diurnal rodent, Octodon degus. Am J Physiol, forthcoming.

Lee TM and Labyak SE (1997) Free-running rhythms and light- and dark-pulse phase response curves for diurnal Octodon degus (Rodentia). Am J Physiol, 273:R278-R286.

Lumia AR, Salchli FJ, Ayers EL, McGinnis MY, McEwan BS, and Teicher MH (1987) Olfactory bulbectomy model of depression: Ejaculatory and circadian dysfunction and their response to antidepressant treatment. Soc Neurosci Abstr 13:1035.

Lumia AR, Teicher MH, Salchli F, Ayers E, and Possidente B (1992) Olfactory bulbectomy as a model for agitated hyposerotonergic depression. Brain Res 587:181-185.
Mick G, Cooper H, and Magnin M (1993) Retinal projection to the olfactory tubercle and basal telencephalon in primates. J Comp Neurol 327:205-219.

Palkovits M, Besson J, and Rotsztejn W (1981a) Distribution of vasoactive intestinal polypeptide in intact, stria terminalis transected and cerebral cortex isolated rats. Brain Res 213:455-459.

Palkovits M, Epelbaum J, and Gros C (1981b) Met-enkephalin concentrations in individual brain nuclei of ansa lenticularis and stria terminalis transected rats. Brain Res 216:203-209.

Pickard GE (1982) The afferent connections of the suprachiasmatic nucleus of the golden hamster with emphasis on the retinohypothalamic projection. J Comp Neurol 211:65-83.

Pieper D and Lobocki C (1991) Olfactory bulbectomy lengthens circadian period of locomotor activity in golden hamsters. Am J Physiol 261:R973-R978.

Possidente B, Lumia AR, McGinnis MY, Rapp M, and McEldowney S (1996) Effects of fluoxetine and olfactory bulbectomy on mouse circadian activity rhythms. Brain Res 713:108-113.

Possidente B, Lumia AR, McGinnis MY, Teicher MH, deLemos E, Sterner L, and Deros L (1990) Olfactory bulb control of circadian activity rhythm in mice. Brain Res 513:325-328.

Scalia F and Winans SS (1975) The differential projections of the olfactory bulb and accessory olfactory bulb in mammals. J Comp Neurol 161:31-56.

Scott JW, Mac Bride RL, and Schneider SP (1980) The organization of projections from the olfactory bulb to the piriform cortex and olfactory tubercle in the rat. J Comp Neurol 194:519-534.

Tessonneaud A, Cooper HM, Caldani M, Locatelli A, and Viguier-Martinez MC (1994) The suprachiasmatic nucleus in the sheep: Retinal projections and cytoarchitectural organization. Cell Tiss Res 278:65-84. 